\title{
Oral Surgery Procedures in a Patient Affected by Hereditary Angioedema Type I
}

\section{Chiara Cinquini (D), Simonetta Santarelli, Alberto Marianelli, Marco Nisi, Mario Gabriele, and Antonio Barone}

\begin{abstract}
Department of Surgical, Medical, Molecular and of Critical Area Pathologies, Complex Operative Unit of Stomatology and Oral Surgery, University-Hospital of Pisa, University of Pisa, 56126 Pisa, Italy
\end{abstract}

Correspondence should be addressed to Antonio Barone; barosurg@gmail.com

Received 11 August 2021; Accepted 19 January 2022; Published 29 January 2022

Academic Editor: Vlaho Brailo

Copyright (c) 2022 Chiara Cinquini et al. This is an open access article distributed under the Creative Commons Attribution License, which permits unrestricted use, distribution, and reproduction in any medium, provided the original work is properly cited.

\begin{abstract}
Hereditary Angioedema (HAE) is a rare disease characterized by a deficiency or a reduced function of the plasma protein $\mathrm{C} 1$ esterase inhibitor (C1-INH), which is involved in the downregulation of several inflammatory pathways. Patients affected by HAE suffer from episodic swellings of subcutaneous or submucosal tissues. Swellings can be caused by stress or dental and surgical procedures and can be life-threatening if the airways are involved. We have reported a clinical case of a patient affected by HAE type I who underwent oral surgery procedures under a short-term prophylaxis with C1-INH plasma-derived concentrate. The patient underwent a cyst removal, multiple tooth extractions, and an excisional biopsy with a prophylaxis with C1-INH plasma-derived concentrate and was hospitalized for 36 hours after the surgery to be monitored for possible HAE attacks. During the hospitalization, the patient did not show signs of swelling nor of HAE attacks. At 14 and 28 days after the surgery, the patient presented a good surgical healing. The prophylactic intravenous infusion of C1-INH concentrate was successful in preventing acute HAE attacks after oral surgery procedures.
\end{abstract}

\section{Introduction}

Hereditary angioedema (HAE) is a rare autosomal dominant disease characterized by a deficiency or a reduced function of the $\mathrm{C} 1$ esterase inhibitor (C1-INH) [1], a plasma protein belonging to the serine protease inhibitors encoded by the SERPING 1 gene [2]. Its incidence ranges from $1 / 50.000$ to $1 / 100.000$, with no distinction of race and sex [3]. Three variants of HAE are portrayed in scientific literature: type I affects $85 \%$ of patients and presents low plasma levels of $\mathrm{C} 1$-INH, and type II affects $15 \%$ of patients and is characterized by inactive $\mathrm{C} 1$-INH with normal plasma levels [4]. HAE with normal C1-INH (HAE-nC1INH) is rare and is characterized by normal $\mathrm{C} 1$-INH level and function and may be associated with mutations in the coagulation factor XII gene or in other known and unknown genes $[5,6]$.
$\mathrm{C} 1$ esterase inhibitor (C1-INH) is involved in the regulation of several inflammatory pathways [1]. Mutations in the gene encoding for the protein C1-INH (SERPING 1 gene) might prevent the synthesis of the protein (HAE type I) or produce a nonfunctioning protein (HAE type II) and both mutations lead to the failure to control the local production of bradykinin. The massive release of this inflammatory mediator increases vascular permeability, thus causing consequent edema of the anatomical areas involved [7]. HAE diagnosis may be suggested by recurrent episodes of swelling and by the presence of abdominal pain [8], even though the final diagnosis can be confirmed only through serological tests [9].

HAE type I is characterized by low plasma levels of C1INH associated with a reduced function and reduced levels of a protein, called complement component 4 (C4), which is a protein belonging to the complement system [10]. 


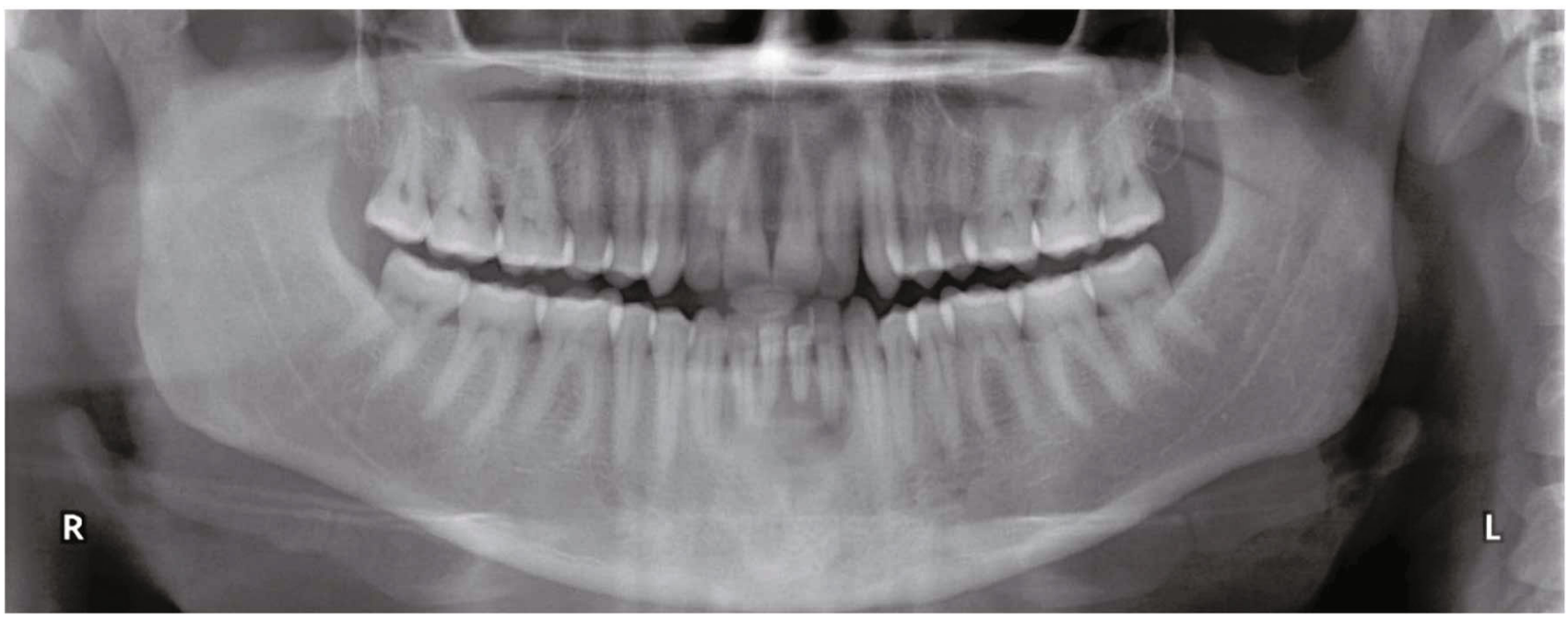

FIgURE 1: Orthopantomogram showing the mandibular radiolucent area.

Some authors showed that the key factors indicating high clinical suspicion for hereditary angioedema are family history and early age in onset of symptoms [8].

Patients affected by HAE suffer from episodic swellings affecting the subcutaneous and submucosal tissues of hands, feet, face, genitals, or gastrointestinal tract [11]; these acute onsets can be life-threatening when the upper airways are involved, especially the larynx, which can cause asphyxia. HAE attacks can be triggered by physical or psychological stress or can arise spontaneously; local trauma such as dental treatments and routine oral surgery procedures may increase the risk of HAE attacks within 4-48 hours from the intervention [12], typically starting near the area of trauma [9]. Several cases of patients' death after dental procedures are described in literature, involving not exclusively patients with recurrent HAE attacks. The mortality rate after oral surgery procedures without adequate prophylaxis was set at $30-40 \%$ in the past decades [13]; at the present time, short-term prophylaxis is used to prevent possible HAE attacks following surgical procedures or other possible triggers [14]. Throughout the years, a considerable number of drugs have been used for prophylaxis before surgical procedures such as C1-INH concentrate, attenuated androgenic hormone therapy, episolon aminocaproic acid, and fresh frozen plasma [12]; C1-INH concentrate has been the most efficient medication because it was developed focusing on the origins of HAE [7].

C1-INH concentrate (plasma-derived or recombinant) has been successfully used to treat acute HAE attacks for many years and, from 2013, has been approved in Europe for shortterm prophylaxis before dental or surgical procedures in patients affected by HAE type I [14]. C1-INH concentrate has a long half-life and can persist in the blood stream for 12 days after the intravenous infusion administration [13].

Other medications approved for the treatment of acute HAE attacks are the kallikrein inhibitors (Ecallantide) and the bradykinin B2 receptor antagonists (Icatibant) $[15,16]$.
Hereditary angioedema is a rare disease that requires special precautions even for common dental or surgical procedures because the consequences might be fatal.

An adjunctive issue is the frequent delayed diagnosis of HAE [17], leading to the effective possibility of having to deal with patients affected by HAE and without being aware of the disease.

This report describes the management of oral surgical intervention in a patient affected by HAE type I.

\section{Case Report}

A 37-year-old man referred to the Unit of Oral Surgery because of episodic swellings in the inferior incisive area. The patient received a complete intraoral examination, showing a complete dentition and a pedunculate lesion of the right buccal mucosa.

During the clinical examination, a diffuse mild swelling was detected at the mandibular buccal incisive area; an orthopantomogram was then prescribed to further investigate the possible origin of the swelling. The radiographic examination revealed a unilocular radiolucency with thin sclerotic margins adjacent to the apical area of mandibular incisors (Figure 1).

Pulp vitality test was negative for teeth 3.1 and 3.2 (lower left incisors), and the teeth were sensitive to percussion. Moreover, teeth 4.1, 3.1, and 3.2 showed a grade III mobility. The suspected diagnosis was inflammatory odontogenic cyst, and the planned surgical intervention was the enucleation of the osteolytic lesion and the evaluation of the involved teeth. Moreover, the biopsy of the pedunculate lesion of the right buccal mucosa was scheduled.

The patient's medical history was collected: since the age of 14, he suffered from four episodes of swelling per year, localized in the hands, feet, face, and genitals. During these episodes, the swelling occurred slowly, involving only one anatomical site. It was associated with a nonpruritic rash and absence of fever, which gradually reduced during the 


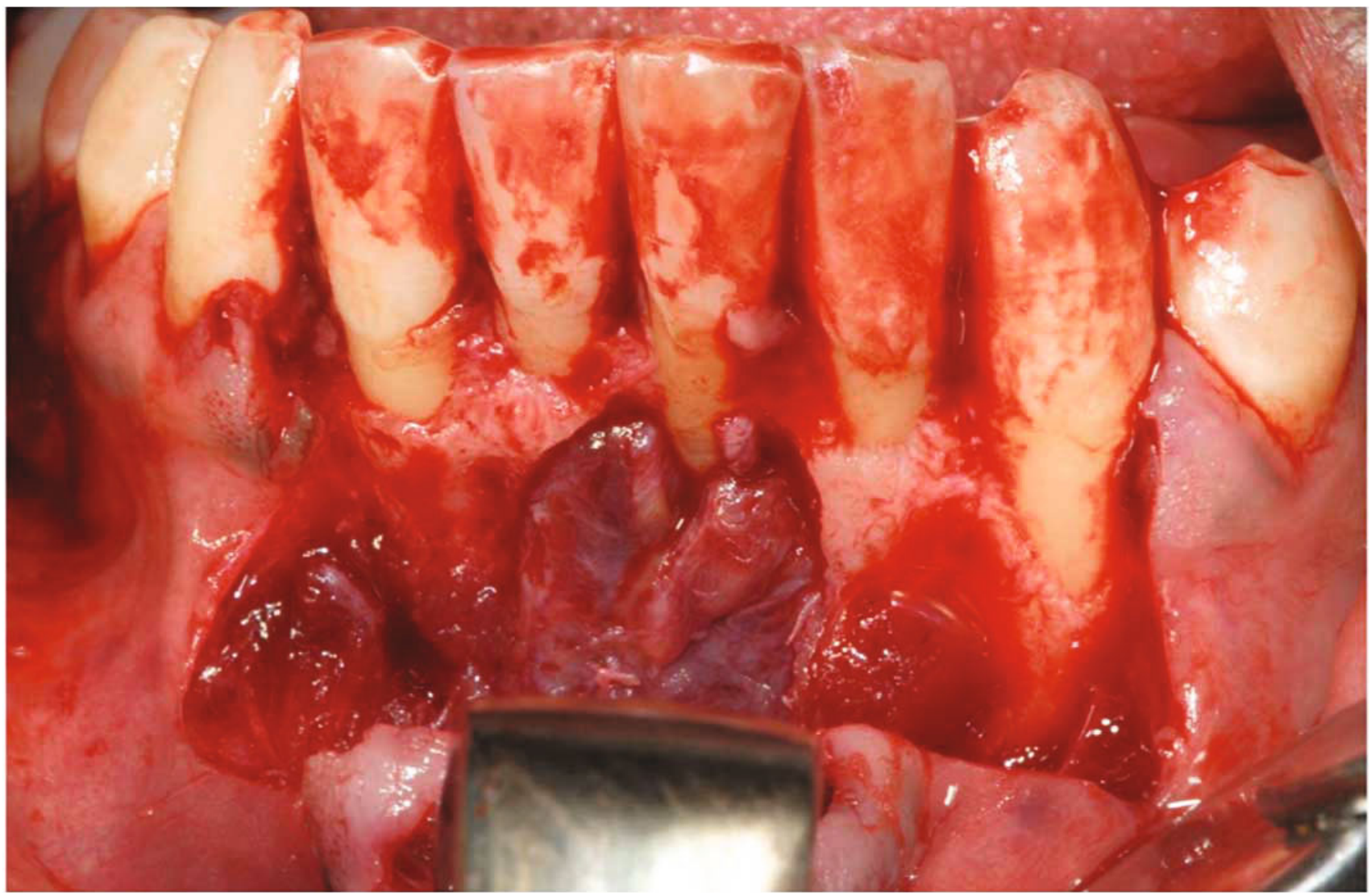

FIGURE 2: Flap elevation and cyst exposure.

following 3-4 days. In the past years, the patient had been hospitalized many times for episodes of abdominal pain and diarrhea. The hematologic analysis for suspected HAE was performed in December 2018: the C1-INH plasmatic concentration was $7.8 \mathrm{mg} / \mathrm{dl}$ (with a normal range between 12 and 30), the functional C1-INH was 34\% (with a normal range between 30 and 130), and $\mathrm{C} 4$ was $7.7 \mathrm{mg} / \mathrm{dl}$ (with a normal range between 10 and 40), confirming the diagnosis of HAE type I.

The medical family history was positive; indeed, his brother, diagnosed with HAE type I, had died from asphyxia due to an HAE attack which occurred after a dental extraction. Complete hematologic analysis was requested a few days before the surgical procedure; erythrocyte, leucocyte, platelet count, prothrombin activity, aPTT and fibrinogen, and coagulation factors were within a normal range.

In agreement with the Immuno-allergology Department, the patient underwent the surgical procedure with a prophylaxis with C1-INH plasma-derived concentrate (Berinert). Berinert is a C1-INH plasma-derived concentrate, usually administered for the short-term prophylaxis of HAE attacks before surgical procedures. The recommended dose of Berinert is $20 \mathrm{IU} / \mathrm{kg}$ (international unit/kilogram), depending on the body weight. On the basis of the patient's weight, 1500 IU were slowly infused intravenously 1 hour before the surgery.

All the surgical procedures were performed under local anesthesia with lidocaine, while monitoring the vital signs (electrocardiography, hearth-rate, blood pressure, and $\mathrm{SpO}_{2}$ ). A mucoperiosteal flap was elevated in the central area of the mandible, revealing a mandibular cyst of approximately $1.5 \times 1.2 \mathrm{~cm}$. The cyst was surgically excised, and three mandibular incisors (4.1,3.1, and 3.2) were extracted due to the extensive bone loss and high mobility (Figures 2 and 3); in fact, both lingual and buccal cortical plates were completely absent once the cyst was removed and the teeth showed a grade III mobility, resulting in an endodonticperiodontal lesion with a hopeless prognosis. The preservation of these teeth could have been resulted in the persistent presence of infection and inflammation, which should be avoided in a HAE patient [4]. An excisional biopsy was also performed to remove and analyze the pedunculate lesion of the right buccal mucosa.

Absorbable sutures were placed to avoid postsurgical removal (Figure 4). Moreover, these sutures made of copolymers of glycolide and lactide are broken down by hydrolysis processes and are not associated with a strong inflammatory reaction [18]. Both surgical specimens were delivered to the Pathological Anatomy Laboratory for the histopathological evaluation. Ropivacaine was instilled in the surgical wound immediately after the surgery to reduce postoperative pain and the consequent stress for the patient.

After the surgery, the patient was asked to report pain using the Numeric Rating Score (NRS) from 0 (no pain at all) to 10 (worst imaginable pain); the NRS value assessed was 6 , and the patient was provided with Tramadol $100 \mathrm{mg}$ 


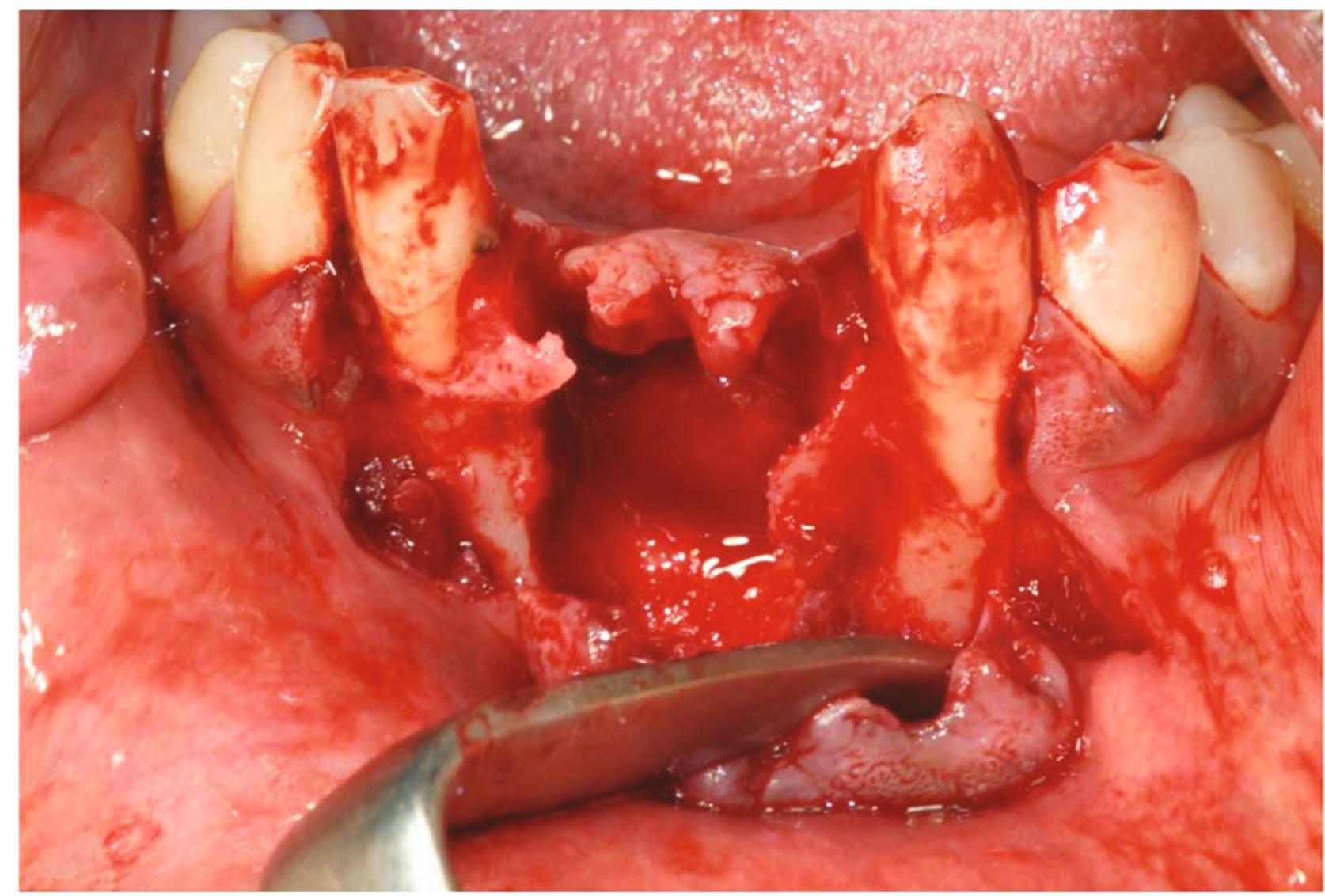

Figure 3: Bone defect after tooth extractions and cyst removal.

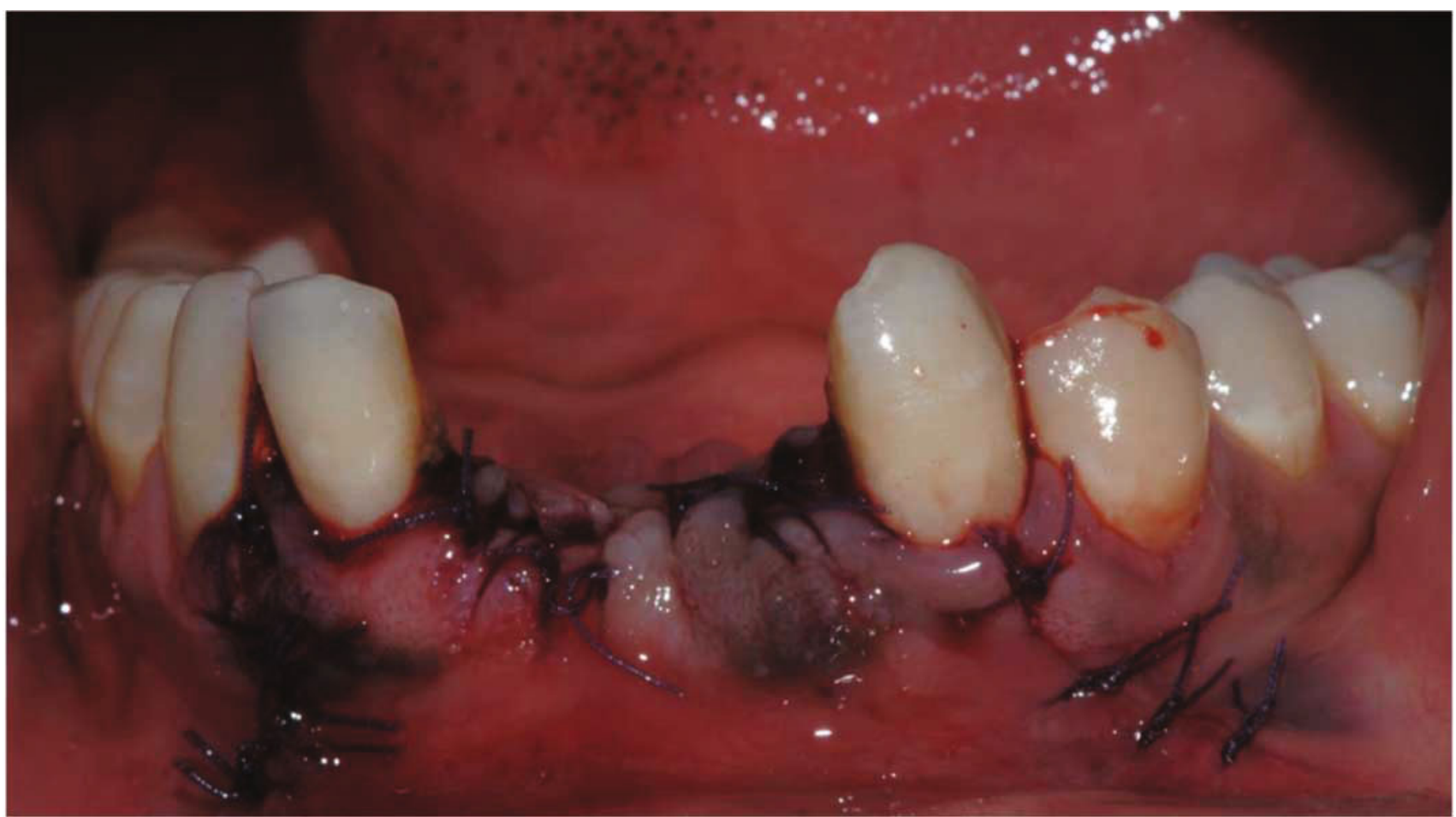

Figure 4: Clinical image immediately after the surgery. 


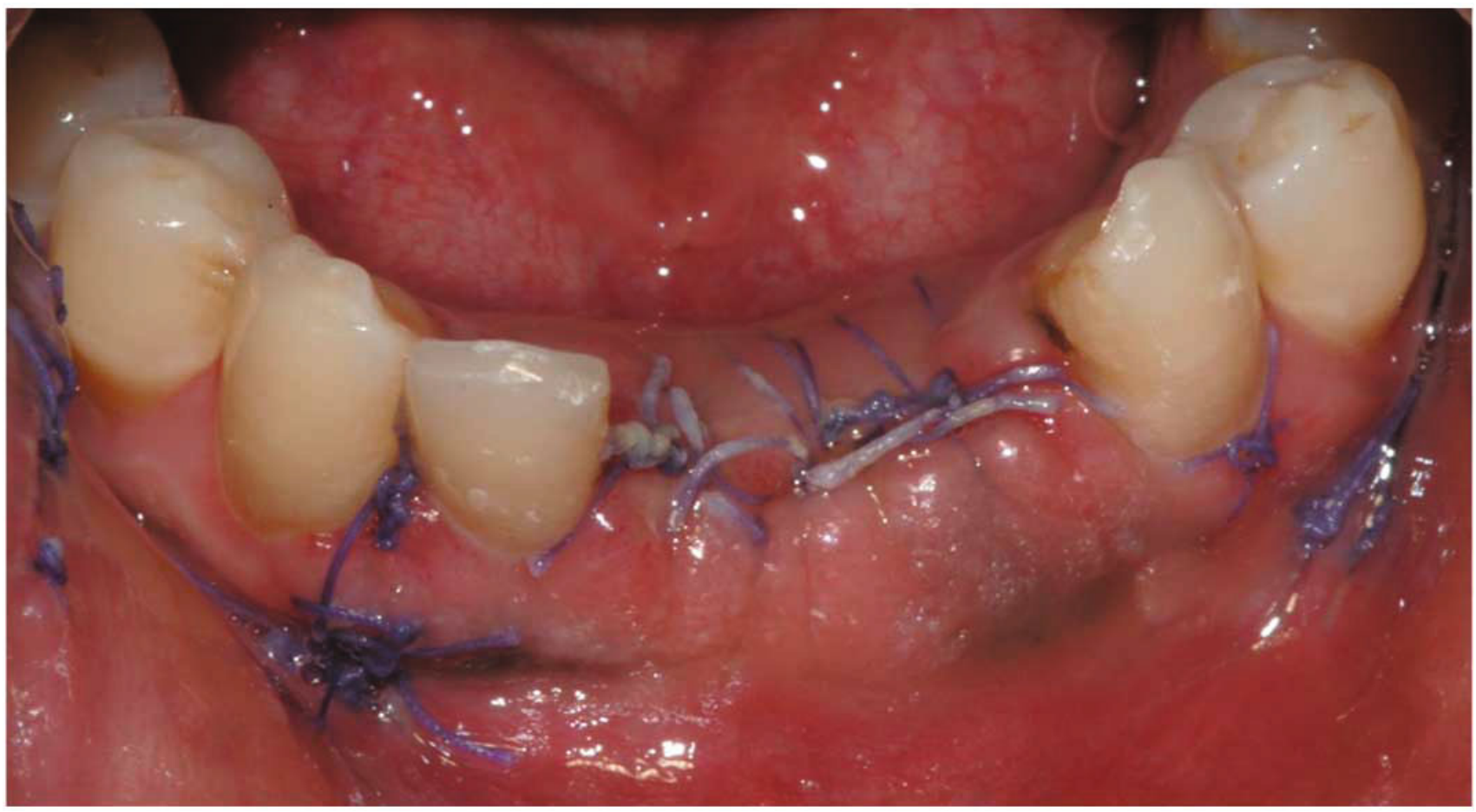

FIgure 5: Clinical image after 14 days from the surgery.

administered intravenously. A postsurgical antibiotic prophylaxis with Clarithromycin was prescribed at the dosage of $500 \mathrm{mg}$ (one tablet) every 12 hours for seven days. Betalactam antibiotics such as amoxicillin were avoided since they may cause drug-induced angioedema [19].

The patient was hospitalized for 36 hours after the surgery to be monitored for possible HAE attacks.

After 36 hours of hospitalization, the patient showed absence of acute HAE episode and he was discharged. He was given exhaustive postsurgical instructions and provided with Icatibant (Firazyr), an antagonist of bradykinin B2 receptors, to be administered subcutaneously by the patient himself in case of an acute attack after the hospitalization period. He was also given the recommendation to go to the emergency department in case of any suspected HAE attacks.

The patient was reevaluated 7 days after the surgery. He had had no occurrence of swelling in the past days and had not required to access the emergency department.

No relevant surgical-related edema was observed. The patient reported no pain $(\mathrm{NRS}=0)$ and no reduction in mouth opening. At the clinical examination, no suppuration or local signs of inflammation were evident.

At 14 (Figure 5) and 28 days after the surgery, the patient presented a good surgical healing and an excellent oral hygiene.

The histopathological findings of the intrabony lesion showed the presence of a fibrous wall with a lymphoplasmacytic and monocytic infiltrate, and a lining epithelium, confirming the clinical diagnosis of inflammatory odontogenic cyst (radicular cyst). With regard to the pedunculate lesion of the buccal mucosa, the presence of sclerotic connective tissue with signs of acanthosis and parakeratosis was compatible with a local fibrous hyperplasia on an irritative basis.

Special attention will be paid for future prosthetic rehabilitation since in literature; there are described acute HAE attacks following impressions for prosthetic rehabilitations [20].

\section{Discussion}

In the past years, the administration of preoperative fresh frozen plasma (FFP) has been an effective method to prevent HAE attacks following dental or surgical procedures [21].

The use of C1-INH concentrate was approved in 2009 in Europe and in 2010 in the USA for the treatment of acute HAE attacks, while its use as prophylactic treatment was finally approved in Europe in 2013.

Many studies in literature reported the use of C1-INH concentrate as prophylaxis before surgical procedures, showing good outcomes.

Maeda et al. [7] described a case report of a patient affected by HAE type I who suffered from swellings after the injection of local anesthetics for routinary dental treatments. The patient required the extraction of a lower third molar, and he was treated under local anesthesia; the infusion of C1-INH was performed just after the surgical procedure. In this case, no postprocedural swelling was observed.

Sanuki et al. [22] reported a successful management of multiple tooth extractions in a patient affected by HAE with the preprocedural administration of the C1-INH 
concentrate. The postoperative period was uneventful, and the patient was discharged home 3 days after the surgery.

A retrospective study on the efficacy of $\mathrm{C} 1$-INH concentrate prophylaxis in preventing HAE attacks showed that out of 24 patients who underwent invasive surgical procedures (varying from dental extractions to aortic aneurysm repair), none of them had a HAE attack [14].

Another retrospective study performed on $24 \mathrm{HAE}$ patients who underwent 66 dental procedures (ranging from dental extraction to orthodontic treatment) showed the efficacy of a preprocedural prophylaxis with C1-INH concentrate or treatment with attenuated androgens (AAs) [11]. According to the authors, only 3 patients experienced a mild upper airway edema; none of these patients received a preprocedural prophylaxis with $\mathrm{C} 1-\mathrm{INH}$ or a maintenance treatment with AAs.

A larger study including 91 patients suffering from $\mathrm{HAE}$ showed that $98 \%$ of the patients, who were treated with $\mathrm{C} 1$ INH concentrate as prophylaxis, had an uneventful healing period after surgical procedures [23].

The International WAO/EAACI (World Allergy Organization and the European Academy of Allergy and Clinical Immunology) guidelines suggest the use of the $\mathrm{C} 1$-INH concentrate as presurgical prophylaxis in patients affected by HAE [10].

The importance of presurgical prophylaxis is confirmed by the fact that HAE attacks do not respond to many antiedema treatments such as antihistamines, corticosteroids, and adrenaline [24].

The prevention of acute attacks is the best course of action in cases of surgical procedures for patients affected by HAE, especially considering the fact that the treatment of acute attacks may be difficult and a potentially lifethreatening complication. However, it should be remembered that acute HAE attacks may occur after relatively minor surgical procedures despite the presurgical prophylaxis [25]; for this reason, patients should be carefully monitored and instructed on what to do at home in case of an acute attack, according to what is reported in the case report section.

In the present case, a presurgical prophylaxis with $\mathrm{C} 1$ INH concentrate was effective in preventing acute $\mathrm{HAE}$ attacks and the postsurgical period was uneventful.

\section{Consent}

The authors declare that the patient has been informed of the publication of this Case Report and signed an appropriate consent form. The patient has given his consent for the publication of anonymized clinical information and radiographic/photographic reports.

\section{Conflicts of Interest}

The authors declare that they have no conflicts of interest.

\section{Authors' Contributions}

All the authors approved the final version of the manuscript and agreed to be accountable for all aspects of the work.

\section{Acknowledgments}

The authors declare that they have no conflict of interest.

\section{Supplementary Materials}

Supplementary file 1: care checklist. (Supplementary Materials)

\section{References}

[1] H. Longhurst and M. Cicardi, "Hereditary angio-oedema," Lancet, vol. 379, no. 9814, pp. 474-481, 2012.

[2] A. E. Germenis and M. Speletas, "Genetics of hereditary angioedema revisited," Clinical reviews in allergy \& immunology, vol. 51, no. 2, pp. 170-182, 2016.

[3] M. S. Gakhal, G. Marcotte, and v., "Hereditary angioedema: imaging manifestations and clinical management," Emergency radiology, vol. 22, no. 1, pp. 83-90, 2015.

[4] T. Caballero, M. L. Baeza, R. Cabañas et al., "Consensus statement on the diagnosis, management, and treatment of angioedema mediated by bradykinin. Part II. Treatment, followup, and special situations," Journal of investigational allergology \& clinical immunology, vol. 21, no. 6, pp. 422-423, 2011.

[5] B. L. Zuraw, "Clinical practice. Hereditary angioedema," The New England journal of medicine, vol. 359, no. 10, pp. 10271036, 2008.

[6] B. L. Zuraw, K. Bork, K. E. Binkley et al., "Hereditary angioedema with normal $\mathrm{C} 1$ inhibitor function: consensus of an international expert panel," Allergy and Asthma Proceedings, vol. 33, no. 6, 2012.

[7] S. Maeda, T. Miyawaki, S. Nomura, T. Yagi, and M. Shimada, "Management of oral surgery in patients with hereditary or acquired angioedemas: review and case report," Oral surgery, oral medicine, oral pathology, oral radiology, and endodontics, vol. 96, no. 5, pp. 540-543, 2003.

[8] M. M. Gompels, R. J. Lock, D. J. Unsworth et al., "Misdiagnosis of hereditary angio-oedema type 1 and type 2," The British journal of dermatology, vol. 148, no. 4, pp. 719-723, 2003.

[9] M. Kunschak, W. Engl, F. Maritsch et al., "A randomized, controlled trial to study the efficacy and safety of $\mathrm{C} 1$ inhibitor concentrate in treating hereditary angioedema," Transfusion, vol. 38, no. 6, pp. 540-549, 1998.

[10] M. Maurer, M. Magerl, I. Ansotegui et al., "The international WAO/EAACI guideline for the management of hereditary angioedema-the 2017 revision and update," Allergy, vol. 73, no. 8, pp. 1575-1596, 2018.

[11] J. Jurado-Palomo, J. M. Muñoz-Caro, M. C. López-Serrano et al., "Management of dental-oral procedures in patients with hereditary angioedema due to $\mathrm{C} 1$ inhibitor deficiency," Journal of investigational allergology \& clinical immunology, vol. 23, no. 1, pp. 1-6, 2013.

[12] N. J. van Sickels, R. B. Hunsaker, and J. E. van Sickels, "Hereditary angioedema: treatment, management, and precautions in patients presenting for dental care," Oral surgery, oral medicine, oral pathology, oral radiology, and endodontics, vol. 109, no. 2, pp. 168-172, 2010.

[13] K. Bork and S.-E. Barnstedt, "Laryngeal edema and death from asphyxiation after tooth extraction in four patients with hereditary angioedema," Journal of the American Dental Association, vol. 134, no. 8, pp. 1088-1094, 1939. 
[14] G. Gavigan, W. H. Yang, S. Santucci, R. Harrison, and J. Karsh, "The prophylactic use of $\mathrm{C} 1$ inhibitor in hereditary angioedema patients undergoing invasive surgical procedures: a retrospective study," Allergy, asthma, and clinical immunology: official journal of the Canadian Society of Allergy and Clinical Immunology, vol. 10, no. 1, p. 17, 2014.

[15] G. Hwang, A. Johri, S. Ng, and T. Craig, "A review of kallikrein inhibitor lanadelumab in hereditary angioedema," Immunotherapy, vol. 11, no. 11, pp. 937-944, 2019.

[16] M. Cicardi, A. Banerji, F. Bracho et al., "Icatibant, a new bradykinin-receptor antagonist, in hereditary angioedema," The New England journal of medicine, vol. 363, no. 6, pp. 532-541, 2010.

[17] M. D. Guarino, C. Perricone, S. Guarino et al., "Denaturing HPLC in laboratory diagnosis of hereditary angioedema," The Journal of allergy and clinical immunology, vol. 120, no. 4, pp. 962-965, 2007.

[18] M. Byrne and A. Aly, "The surgical suture," Aesthetic surgery journal, vol. 39, Supplement_2, pp. S67-S72, 2019.

[19] N. Inomata, "Recent advances in drug-induced angioedema," Allergology International, vol. 61, pp. 545-557, 2012.

[20] S. R. Aziz and P. Tin, "Spontaneous angioedema of oral cavity after dental impressions," The New York state dental journal, vol. 68, no. 2, pp. 42-45, 2002.

[21] J. C. Atkinson and M. M. Frank, "Oral manifestations and dental management of patients with hereditary angioedema," Journal of Oral Pathology \& Medicine, vol. 20, no. 3, pp. 139-142, 1991.

[22] T. Sanuki, T. Watanabe, S. Kurata, and T. Ayuse, "Perioperative management of tooth extractions for a patient with hereditary angioedema," Journal of Oral and Maxillofacial Surgery, vol. 72, no. 2421, pp. e1-2421.e24213, 2014.

[23] J. A. Grant, M. White, H. H. Li et al., "Preprocedural administration of nanofiltered $\mathrm{C} 1$ esterase inhibitor to prevent hereditary angioedema attacks," Allergy and asthma proceedings, vol. 33, no. 4, pp. 348-353, 2012.

[24] B. L. Zuraw, "Current and future therapy for hereditary angioedema," Clinical immunology (Orlando, Fla)., vol. 114, no. 1, pp. 10-16, 2005.

[25] E. Aygören-Pürsün, I. Martinez Saguer, W. Kreuz, T. Klingebiel, and D. Schwabe, "Risk of angioedema following invasive or surgical procedures in HAE type I and II- the natural history," Allergy, vol. 68, no. 8, 2013. 